NASA-TM-104,037

NASA Technical Memorandum 104037

NASA-TM-104037 19910021825

\title{
A TECHNIQUE FOR LOCATING FUNCTION ROOTS AND FOR SATISFYING EQUALITY CONSTRAINTS IN OPTIMIZATION
}

Jaroslaw Sobieszczanski-Sobieski

August 1991

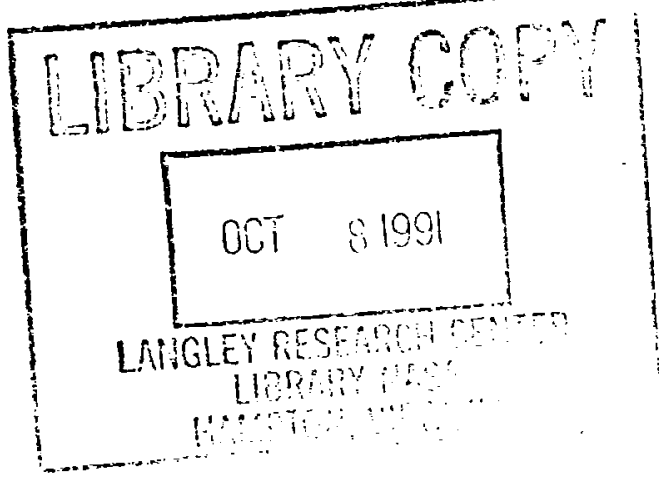

NNSA

National Acronautıcs and

Space Administration

Langley Research Center

Hampton. Virginia 23665-5225 


\title{
A 'TECHNIQUE FOR IOCA'TING FUNC'IION ROOTS AND FOR SATISFYING HQUALITIY CONSTRAIN'I'S IN OP'TIMIZATION
}

\author{
Jaroslaw Sobieszczannki-Sobieski
}

August 1991

\section{Summary}

A new technique for locating simultaneous roots of a set of functions is described. The technique is based on the property of the Kreissclmeier-Stcinhauser function which descends to a minimum at each root location. It is shown that the ensuing algorithm may be merged into any nonlinear programming method for solving optimization problems with equality constraints.

\section{Introduction}

The problem of locating function roots, and the related problem of satisfying equality constraints, arises often in engineering numerical analysis and optimization. In multifunction, multi-variable applications this problem tends to cause numerical difficulties, detrimental to both accuracy and computational efficiency. It is, therefore, of interest to note a new alternative for solution of the above problem based on the properties of a function that became known as the Kreisselmeier-Steinhauser function after it was introduced in reference 1. The present paper defines the new, alternative algorithm and describes its applications as a tool for finding the function roots, and for solving nonlinear programming optimization problems with equality constraints.

\section{The KS Function and Its Properties Useful for Root-Finding}

The algorithm is based on the Kreisselmeier-Steinhauser function ( $K S$ function). The $K S$ function is a differentiable envelope function for a set of functions of the form $Y=F_{k}(X)$, $k=1 \ldots N K$. It is assumed that each of the above functions is continuous in $X$, but not necessarily continuous in its derivatives with respect to $X$. The $K S$ function is expressed in two alternative but completely equivalent formats

$$
\begin{gathered}
K S\left(F_{k}\right)=(1 / \rho) \ln \left(\sum_{k} \exp \left(\rho F_{k}\right)\right), \quad k=1 \ldots N K \\
K S\left(F_{k}\right)=F_{\max }+(1 / \rho) \ln \left(\sum_{k} \operatorname{cxp}\left(\rho\left(F_{k}-F_{\max }\right)\right)\right), \quad k=1 \ldots N K
\end{gathered}
$$

'The format in equation (2) is recommended if the standard format in equation (1) generates too large values of the exponential function. 
The $K S$ function has a property that

$$
F_{\max } \leq K S\left(F_{k}\right) \leq F_{\max }+\ln (N K) / \rho, \quad k=1 \ldots N K
$$

where $\rho$ is a user-controlled parameter.

Example of the $K S$ function is plotted in figure 1 for one independent variable and a set of three functions. As implied by equation (3), the user may draw $K S$ closer to $F_{\max }$ by increasing the value of parameter $\rho$ and vice versa. In other words, the $\rho$ parameter is a means for controlling how close the $K S$ follows the piecewise envelope of the set of functions $F_{k}$. The $K S$ function is a differentiable equivalent of the non-differentiable "selective" function $\operatorname{MAX}\left(F_{k}\right)$ available in many high-level programming languages.

The $K S$ function derivatives may also be expressed in two ways, corresponding to equations (1) and (2), respectively,

$$
\begin{gathered}
\frac{\partial K S}{\partial X_{i}}=\left(\sum_{k}\left(\exp \left(\rho F_{k}\right)\right) \frac{\partial F_{k}}{\partial X_{i}}\right) / \sum_{k} \exp \left(\rho F_{k}\right) \\
\frac{\partial K S}{\partial X_{i}}=\frac{\partial F_{\max }}{\partial X_{i}}+\left(\sum_{k} \exp \left(\rho\left(F_{k}-F_{\max }\right)\right) \frac{\partial F_{k}}{\partial X_{i}}\right) / \sum_{k} \exp \left(\rho\left(F_{k}-F_{\max }\right)\right)
\end{gathered}
$$

The $K S$ function may be used as a tool for root finding because it has the following property, defined using $Z$ as a generic, single independent variable:

"If a function $F_{k}(Z)$ has a root $F_{k}(Z)=0$ for $Z=Z_{r}$, the $K S$ function of $F_{k}$ and $-F_{k}$ is at minimum at $Z=Z_{r}$ for any value of nonzero and nonnegative $\rho . "$

The above property is illustrated in figure 2 and may be proven as a theorem as follows. The property requires that the first derivative of $K S\left(F_{k}(Z),-F_{k}(Z)\right)=$ $(1 / \rho) \ln \left(\exp \left(\rho F_{k}\right)+\exp \left(-\rho F_{k}\right)\right)$ with respect to $Z$ vanish at $Z=Z_{r}$. Considering that

$$
\frac{\partial\left(-F_{k}\right)}{\partial Z}=-\frac{\partial F_{k}}{\partial Z}
$$

differentiation of $K S\left(F_{k},-F_{k}\right)$ yields

$$
\frac{\partial K S}{\partial Z}=\left(\exp \left(\rho F_{k}\right) \frac{\partial F_{k}}{\partial Z}-\exp \left(-\rho F_{k}\right) \frac{\partial F_{k}}{\partial Z}\right) /\left(\exp \left(\rho F_{k}\right)+\exp \left(-\rho F_{k}\right)\right)
$$
Since at $Z=Z_{r}, F_{k}=0$ so that $\exp \left(\rho F_{k}\right)=\exp \left(-\rho F_{k}\right)=1$ regardless of the value of $\rho$,
it follows that

$$
\frac{\partial K S}{\partial Z_{r}}=0
$$

which proves the theorem. Generalization of the above to a function with several roots shows that the corresponding $K S$ would have multiple minima.

Extension to a set of functions $F_{k}(Z), k=1 \ldots N K$, in a hyperspace of $N N$ dimensions ( $Z$ is then a vector of length $N N)$ is straightforward owing to the property represented by equation (3). The previous theorem extended to the above general case is: 
"The $K S$ function of a function set made up of the original set of $F_{k}(Z)$ functions and their negatives, $-F_{k}(Z)$, (mirror images),

$$
\begin{aligned}
K S(Z)= & K S\left(F_{1}(Z), \quad-F_{1}(Z), \quad F_{2}(Z),\right. \\
& \left.-F_{2}(Z), \quad \ldots F_{k}(Z), \quad-F_{k}(Z), \quad \ldots F_{N K}(Z), \quad-F_{N K}(Z)\right)
\end{aligned}
$$

has a local minimum at each point in the $Z$ hyperspace where all the functions $F_{k}(Z)$ attain zero value simultaneously."

The above can be proven by observing that differentiation of $K S$ in equation (9) yields a derivative expression similar to equation (7) extended to include derivatives of all the $\left(+F_{k},-F_{k}\right)$ pairs, while equations (6) and (8) hold for each pair of derivatives. Thus, each point where $K S$ attains minimum defines a solution $Z_{r}$ to a set of simultaneous equations

$$
F_{k}\left(Z_{i}\right)=0 ; \quad k=1 \ldots N K ; \quad i=1 \ldots N N
$$

\section{Numerical Example}

A numerical example illustrating the above is a set of two functions of two variables $u$ and $v$ :

$$
\begin{aligned}
& f_{1}=-1+(u-3)^{2}+(v-2)^{2} \\
& f_{2}=-1+(u-2)^{2}+(v-1)^{2}
\end{aligned}
$$

whose root locus plots in the $u, v$ plane, shown in figure 3 , are circles centered at the points $(3,2)$ and $(2,1)$, respectively. One solution of the set of two simultaneous equations $f_{1}=0$, $f_{2}=0$ is the intersection point of the two circles at $(2,2)$. The values of $K S$ at that point and four neighboring points, $(1.9,2),(2.1,2),(2,1.9)$, and $(2,2.1)$, are given in table 1 for $\rho=50$ :

Table 1. The $K S$ values for $\rho=50$ at, and in, the neighborhood of the intersection point $(2,2)$

\begin{tabular}{|c|c|c|c|}
\hline$u$ & 1.9 & 2.0 & 2.1 \\
\hline$v$ & & & \\
1.9 & .2225386 & .1900034 & .1938629 \\
2.0 & .2100012 & .0277259 & .1900034 \\
2.1 & .2338629 & .2100012 & .2225386 \\
\hline
\end{tabular}


Table 2 shows similar data for the other solution point, the intersection point $(3.0,1.0)$.

Table 2. The $K S$ values for $\rho=50$ at, and in, the neighborhood of the intersection point $(3,1)$

\begin{tabular}{|c|c|c|c|}
\hline$u$ & 2.9 & 3.0 & 3.1 \\
\hline$v$ & & & .2338629 \\
1.0 & .2225386 & .2100012 & .2100012 \\
1.1 & .1900034 & .0277259 & .2225386 \\
\hline
\end{tabular}

Both tables show well-defined minima of $K S$, the underscored values, at both solution (intersection) points.

\section{The Root Finding Algorithm}

Given the above $K S$ function theorem, one may find a root of a set of functions $F_{k}(X)$ by solving the following unconstrained optimization problem

$$
\begin{aligned}
& \text { "find } X \text { such that the objective function } K S\left(F_{k}(X),-F_{k}(X)\right), \\
& \quad k=1 \ldots N K \text {, is at minimum" }
\end{aligned}
$$

Solution of the above problem will identify an $X=X_{o}$ that renders all $F_{k}\left(X_{o}\right)=0$, $k=1 \ldots N K$, simultaneously. This problem may be solved by any technique of Nonlinear Mathematical Programming (NLP). The caveat is that a single execution of such technique will locate only one of, possibly, many different roots as a local minimum of the $K S$ function. Fortunately, in engineering applications, the interest is usually limited to a few roots, and the subdomains containing these roots may be estimated by conjecture. Consequently, repetition of the optimization in equation (11) starting from initial points judiciously chosen in each such domain may be used to locate all the roots of interest.

\section{Satisfaction of Equality Constraints} as

A standard optimization problem with inequality and equality constraints may be written

"find $X_{\text {opt }}$ such that objective function $f\left(X_{\text {opt }}\right)$

is at minimum subject to constraints:

$$
\begin{gathered}
g_{j}\left(X_{\mathrm{opt}}\right) \leq 0, \quad j=1 \ldots M G ; \\
h_{p}\left(X_{\mathrm{opt}}\right)=0, \quad p=1 \ldots M H ;
\end{gathered}
$$

Using the $K S$ theorem introduced in the aforegoing, one may convert the above problem into one that includes only the inequality constraints 
"find $X_{\text {opt }}$ such that objective function $f\left(X_{\text {opt }}\right)$ is at minimum subject to constraints:

$$
\begin{aligned}
g_{j}\left(X_{\mathrm{opt}}\right) \leq 0, \quad j & =1 \ldots M G \\
K S\left(h_{p}\left(X_{\mathrm{opt}}\right), \quad-h_{p}\left(X_{\mathrm{opt}}\right)\right) & \leq T O L, \quad p=1 \ldots M H ; "
\end{aligned}
$$

where TOL is a suitably small, positive tolerance. Any NLP technique may be used to determine $X_{\text {opt }}$ in the above problem that is, in general, easier to solve than the one in equation (12) because it includes no equality constraints.

One may note that the $K S$ function in equation (13b) is an extension of a cumulative constraint concept that was successfully used in conjunction with inequality constraints in many applications, e.g., reference 2 , whereby a single $K S$ function represented a number of constraints. Extending that concept further, both the inequality and equality constraints may be blended into one cumulative constraint, so that equation (13) transforms into

$$
\begin{aligned}
& \text { "find } X_{\text {opt }} \text { such that objective function } f\left(X_{\mathrm{opt}}\right) \\
& \text { is at minimum subject to constraints: }
\end{aligned}
$$

$$
\begin{aligned}
& K S\left(g_{j}\left(X_{\mathrm{opt}}\right), \quad h_{p}\left(X_{\mathrm{opt}}\right), \quad-h_{p}\left(X_{\mathrm{opt}}\right)\right) \leq T O L, \\
& j=1 \ldots M G, \quad p=1 \ldots M H ; "
\end{aligned}
$$

Again, the presence of $h$-constraints in the above may add to the number of the local optima that might already be present in the same problem with the inequality constraints only. Under the present state-of-the-art, there is no guaranteed way for locating the global minimum in this class of problems, except to repeat the optimization starting from several judiciously chosen initial design points.

\section{Conclusions}

A particular property of the Kreisselmeier-Steinhauser function known for its use as a cumulative constraint was identified as useful for locating roots of a set of functions. Based on that property, an algorithm was formulated for solving a general, nonlinear programming problem with inequality and equality constraints by transforming it into one with inequality constraints only. The transformed problem may be solved by any NLP technique capable of solving inequality constrained problems. 


\section{References}

1. Kreisselmeier, G.; and Steinhauser, R.: Application of Vector Performance Optimization to a Robust Control Loop Design for a Fighter Aircraft. International Journal of Control, Vol. 37, No. 2, 1983, pp. 251-284.

2. Sobieszczanski-Sobieski, J.; James, B. B.; and Riley, M. F.: Structural Sizing by Generalized Multilevel Optimization. AIAA J., Vol. 25, No.1, January 1987, pp. 139-145. 


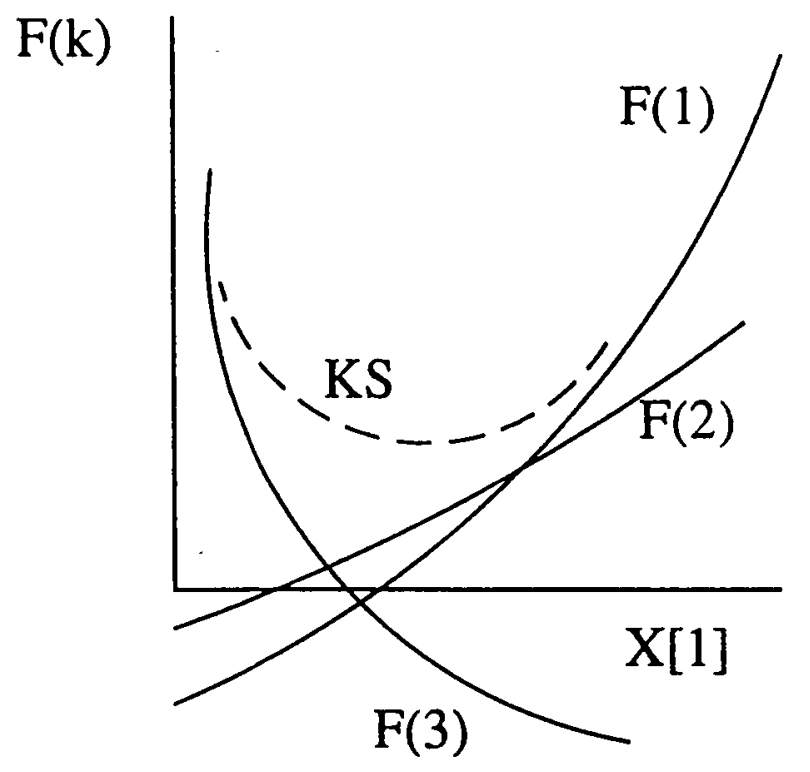

Fig. $1 \mathrm{KS}$ function as envelope of a set of functions

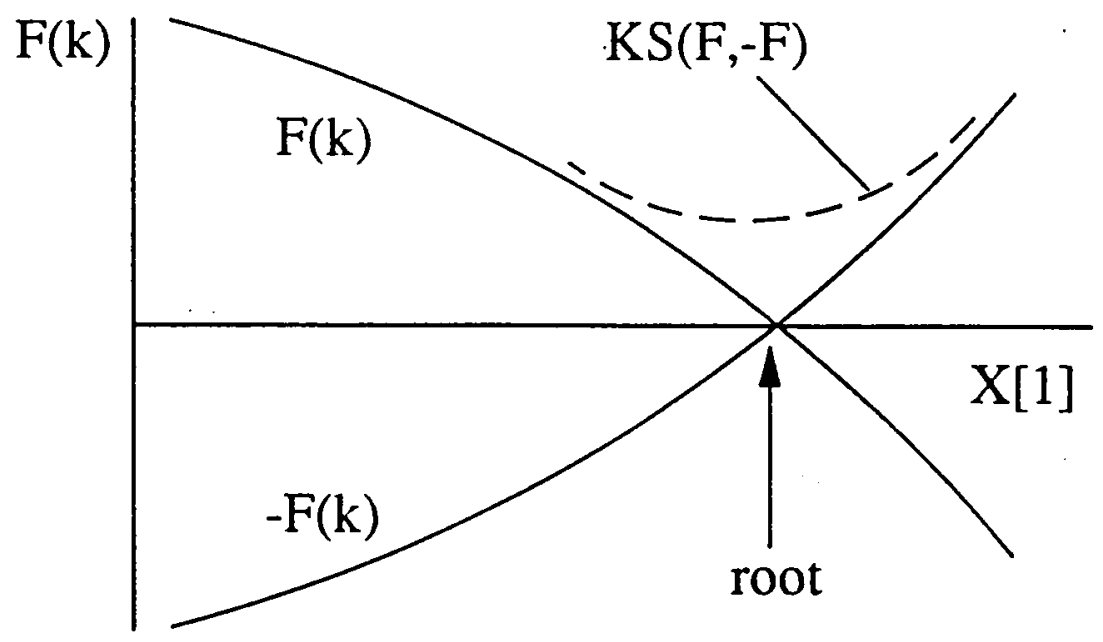

Fig.2 KS minimum coinciding with a root of function

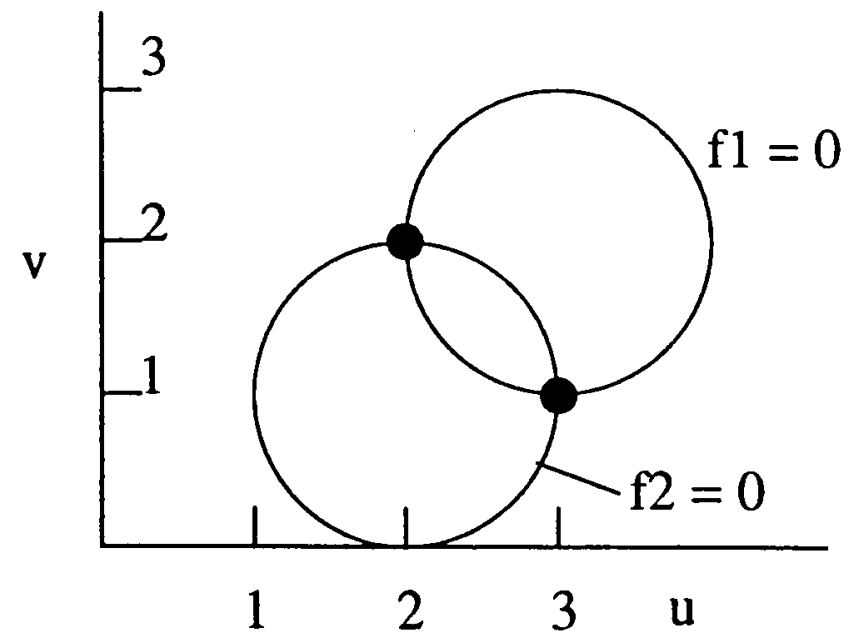

Fig.3 Solution points of simultaneous nonlinear equations 


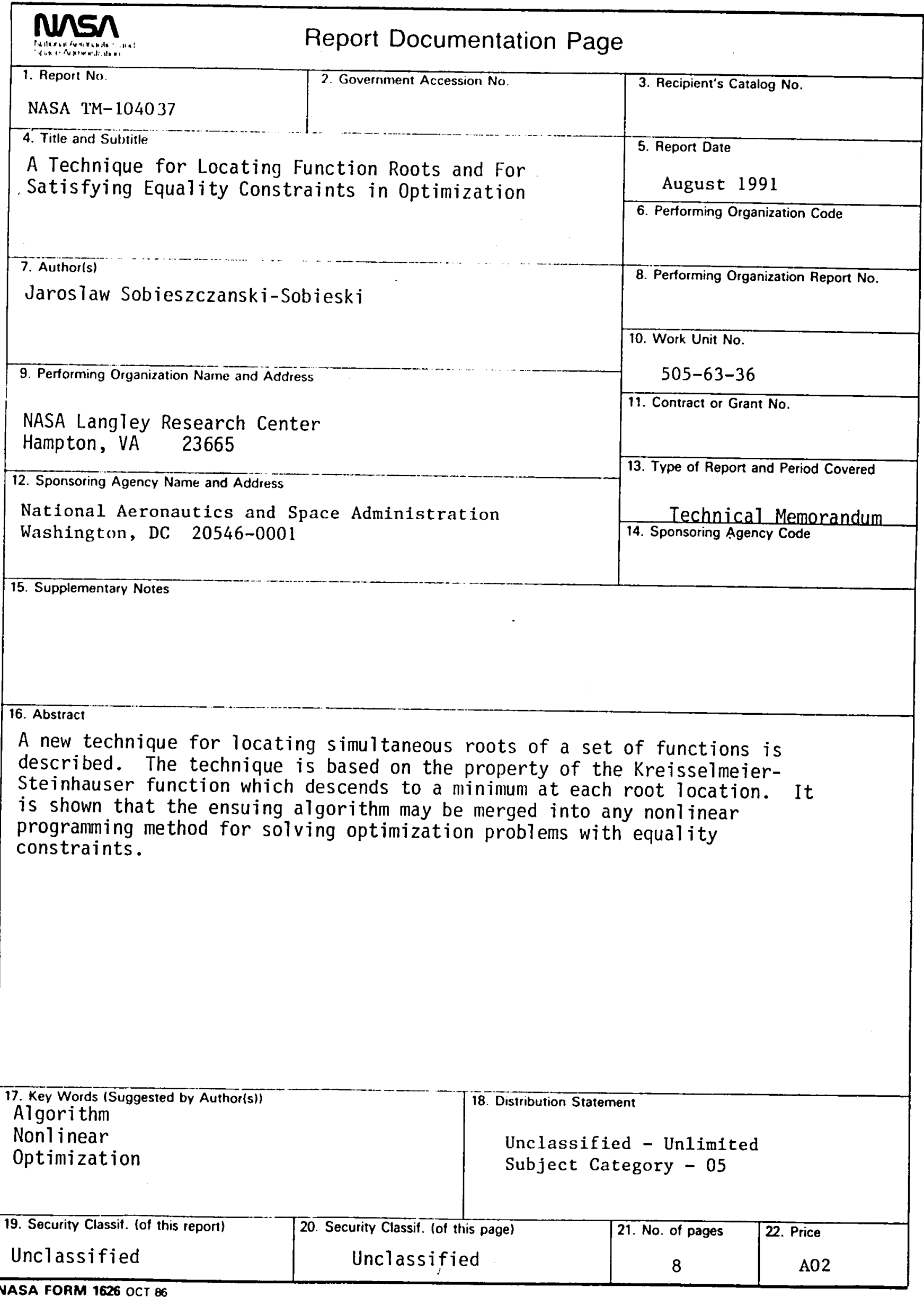


. 
. 